\title{
Color Preferences of Patients Receiving Elastic Ligatures
}

\author{
Selma Elekdag-Turk \\ Nurhat Ozkalayci ${ }^{b}$ \\ Devrim Iscia \\ Tamer Turk ${ }^{c}$
}

\begin{abstract}
Objectives: To determine the color preferences for elastic ligatures among patients receiving fixed-appliance orthodontic therapy.

Methods: Five hundred patients with metal brackets (336 female patients and 164 male patients) were included, and their color preferences for elastic ligatures were recorded using a color scale. Chi-square analysis was performed to evaluate the color preferences and to determine the influence of age and gender on color preferences $(P<.05)$.

Results: Two hundred and seventy-six patients (55.2\%) preferred strikingly colorful elastic ligatures, while 224 patients (44.8\%) preferred less noticeable elastic ligatures. No significant difference was observed between the color preferences of female and male patients ( $P>05$ ). However, significant differences were observed between the color preferences of adolescents and older patients $(P<.001)$.

Conclusions: Female patients preferred red-purple-colored tones, while male patients preferred blue-black-colored tones. Adolescents preferred colorful elastic ligatures, while older patients preferred ligatures with less-noticeable colors. A stock of 10-12 colorful and less-noticeable elastic ligatures seems to be adequate to ensure patient satisfaction. (Eur J Dent 2010;4:171-174)
\end{abstract}

Key words: Color preference; Elastic ligatures; Orthodontic therapy.

n- Assistant Professor, Department of Orthodontics, Faculty of Dentistry, University of Ondokuz Mayis, Samsun, Turkey.

${ }^{\mathrm{b}}$ Research Assistant, Department of Orthodontics, Faculty of Dentistry, University of Ondokuz Mayis, Samsun, Turkey.

'Professor, Department of Orthodontics, Faculty of Dentistry, University of Ondokuz Mayis, Samsun, Turkey.

- Corresponding author: Dr. Selma Elekdag-Turk University of Ondokuz Mayis, Faculty of Dentistry, Department of Orthodontics, 55139 Kurupelit/Samsun, Turkey. Phone: +90 5446403424 Fax: +90 3624576032 E-mail: turksetđsuperonline.com

\section{INTRODUCTION}

Elastic ligatures are commonly used in fixedappliance orthodontic treatment. ${ }^{1}$ Archwire ligation performed using elastic ligatures is faster than that performed using wire ligatures. Moreover, the availability of elastic ligatures with different colors facilitates active patient involvement in the treatment process, which may have a positive influence on patient cooperation during treatment.

However, no information is available on patients' color preferences for elastic ligatures. Which colors of elastic ligatures should orthodon- 
tists stock and present to their patients? A comment on this subject has suggested that about 10 colors should be stocked at all times.'

To elucidate this aspect of fixed-appliance orthodontic therapy, the color preferences for elastic ligatures among patients receiving fixed orthodontic appliances were determined and the influence of age and gender on these preferences was evaluated.

\section{MATERIALS AND METHODS}

Five hundred patients (336 female patients and 164 male patients) were included in this study. Patients who had received metal brackets and were at least in the $4^{\text {th }}$ month of fixed-appliance orthodontic treatment were included, because, in comparison with the patients in the earlier stages of treatment, these patients were more experienced in choosing ligatures. The color preferences of the patients were recorded using a color scale available on the market (American Orthodontics, Sheboygan, WI, U.S.A.) (Figure 1). One preferred color was recorded for each patient.

Transparent ligatures and ligatures with colors such as white, pearl, and silver were defined as

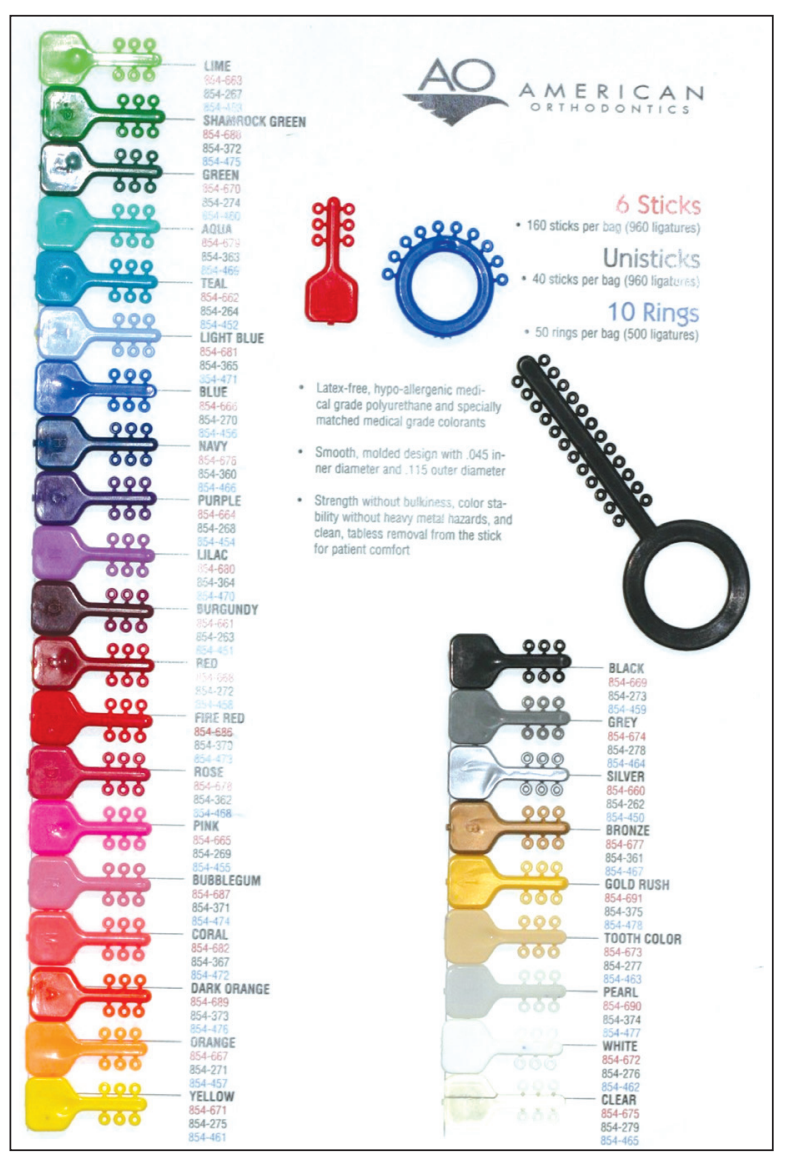

Figure 1. Color scale. "less noticeable," while ligatures with more striking colors such as pink, yellow, and purple were defined as "colorful."

Chi-square analysis was performed to evaluate the color preferences and to determine the influence of age and gender on color preferences $(P<.05)$.

\section{RESULTS}

The age and gender distributions of the patients are provided in Table 1. Two hundred and seventy-six patients (55.2\%) preferred colorful elastic ligatures, while 224 (44.8\%) chose lessnoticeable elastic ligatures (Table 2).

The distribution of color preferences among the patients who chose colorful elastic ligatures is as follows: pink, 59 (21.4\%); light blue, 34 (12.3\%); blue, 33 (12.0\%); lilac, 29 (10.5\%); fire red, 24 $(8.7 \%)$; lime, 20 (7.2\%); black, $20(7.2 \%)$; yellow, $11(4.0 \%)$; and purple, 11 (4.0\%) (Table 3). The distribution of color preferences among the patients who chose less-noticeable elastic ligatures is as follows: transparent, 184 (82.1\%); white, 19 (8.5\%); silver, 17 (7.9\%); and pearl, 4 (1.8\%) (Table 4).

The relationship between gender and color preference is presented in Table 5 . No significant difference was observed between the color preferences of female and male patients ( $P>$.05). However, significant differences were observed between the color preferences among patients of different age groups (Table 6; $P<.001$ ); older patients preferred elastic ligatures with less-notice-

Table 1. Characteristics of the patients.

\begin{tabular}{lcc}
\hline & Number & Percentage \\
\hline Number of patients & 500 & - \\
Gender-related distribution & & \\
Female & 336 & 67.2 \\
Male & 164 & 32.8 \\
Age-related distribution & & \\
$\leq 15$ & 197 & 39.4 \\
$16-20$ & 228 & 45.6 \\
$\geq 21$ & 75 & 15.0 \\
\hline
\end{tabular}

Table 2. Distribution of color preference of patients.

\begin{tabular}{lcc}
\hline & Number & Percentage \\
\hline Colorful & 276 & 55.2 \\
Less-noticeable & 224 & 44.8 \\
\hline
\end{tabular}


able colors. The detailed age- and gender-wise distributions of color preferences are shown in Tables 7 and 8.

\section{DISCUSSION}

Gender has been shown to influence color preferences. ${ }^{2}$ While females prefer red-purplecolored tones, males prefer blue-green-colored tones. ${ }^{2}$ Neuroscientists Hurlbert and Ling ${ }^{2}$ supported the view that color preferences among the sexes have a biological basis, i.e., an evolutionary basis, rather than a cultural basis. The results of Alexander's study ${ }^{3}$ implied that females prefer reds since their eyes are attuned to perceive reds better than the other colors. This finding supports Hurlbert and Ling's idea of an evolutionary basis for color preferences. Nevertheless, researchers at Princeton University suggest that color preferences are influenced by a combination of biological and cultural biases. ${ }^{4}$
However, in the present study, the differences between the color preferences of female and male patients were not significant. While female patients showed a slightly higher preference for colorful elastic ligatures, male patients showed identical preferences for colorful and less-noticeable elastic ligatures. Among the ligatures with less-noticeable colors, transparent ligatures were preferred by both female and male patients. In fact, the preferences for transparent ligatures were almost equal among female and male patients: $36.3 \%$ for female patients and $37.9 \%$ for male patients. Detailed analysis of the preferences for colorful ligatures showed that female patients usually preferred lilac, pink, lime, purple, and light blue ligatures. In contrast, male patients usually preferred blue and black ligatures.

Another notable finding in this study was that fire-red ligatures were chosen by both female and male patients. While the preference for red

Table 3. Color distribution of colorful elastic ligatures.

\begin{tabular}{lccc}
\hline Color & Number & Percentage (colorful ligatures) & Percentage (all elastic ligatures) \\
\hline Fire Red & 24 & 8.7 & 4.8 \\
Lilac & 29 & 10.5 & 5.8 \\
Yellow & 11 & 4.0 & 2.2 \\
Blue & 33 & 12.0 & 6.6 \\
Pink & 59 & 21.4 & 11.8 \\
Lime & 20 & 7.2 & 4.0 \\
Purple & 11 & 4.0 & 2.2 \\
Black & 20 & 7.2 & 4.0 \\
Light Blue & 34 & 12.3 & 6.8 \\
Various & 35 & 12.7 & 7.0 \\
\hline
\end{tabular}

Table 4. Color distribution of less-noticeable elastic ligatures.

\begin{tabular}{lccc}
\hline Color & Number & Percentage (colorful ligatures) & Percentage (all elastic ligatures) \\
\hline Transparent & 184 & 82.1 & 36.8 \\
White & 19 & 8.5 & 3.8 \\
Pearl & 4 & 1.8 & 0.8 \\
Silver & 17 & 7.9 & 3.4 \\
\hline
\end{tabular}

Table 5. Relationship of gender and color preference.*

\begin{tabular}{lcc}
\hline \multirow{2}{*}{ Elastic ligature } & \multicolumn{2}{c}{ Gender } \\
& Female & Male \\
\hline Colorful & $194(57.7 \%)$ & $82(50.0 \%)$ \\
Less-noticeable & $142(42.3 \%)$ & $82(50.0 \%)$ \\
\hline
\end{tabular}

* Percentages show the distribution for each gender.

Table 6. Relationship of age and color preference.*

$\chi^{2}=2.668, P=0.102$

\begin{tabular}{lccc}
\hline Elastic & \multicolumn{3}{c}{ Age } \\
ligature & $\leq 15$ & $16-20$ & $\geq 21$ \\
\hline Less-noticeable & $55(27.9 \%)$ & $112(49.1 \%)$ & $57(76.0 \%)$ \\
Colorful & $142(72.1 \%)$ & $116(50.9 \%)$ & $18(24.0 \%)$ \\
\hline
\end{tabular}

* Percentages show the distribution for each age group. $\chi^{2}=53.947, P<.001$ 
among female patients has been explained, the preference for red among male patients can be attributed to the association of the color with their favorite football teams.

Detailed analysis in terms of the age of the patients revealed a high preference for colorful ligatures among adolescents. Almost none of adults (age, 21 years and higher) preferred color-

Table 7. Detailed gender-related distribution of elastic ligature preferences.*

\begin{tabular}{lcc}
\hline Color & Female & Male \\
\hline Fire red & $13(3.9 \%)$ & $11(6.7 \%)$ \\
Lilac & $28(8.3 \%)$ & $1(0.6 \%)$ \\
Yellow & $3(0.9 \%)$ & $8(4.9 \%)$ \\
Blue & $9(2.6 \%)$ & $24(14.6 \%)$ \\
Pink & $59(17.6 \%)$ & $0(0.0 \%)$ \\
Lime & $15(4.5 \%)$ & $5(3.0 \%)$ \\
Purple & $10(3.0 \%)$ & $1(0.6 \%)$ \\
Black & $5(1.5 \%)$ & $15(9.1 \%)$ \\
Light Blue & $28(8.3 \%)$ & $6(3.7 \%)$ \\
Transparent & $122(36.3 \%)$ & $62(37.9 \%)$ \\
White & $9(2.6 \%)$ & $10(6.1 \%)$ \\
Pearl & $3(0.9 \%)$ & $1(0.6 \%)$ \\
Silver & $8(2.4 \%)$ & $9(5.5 \%)$ \\
Various & $24(7.2 \%)$ & $11(6.7 \%)$ \\
\hline
\end{tabular}

* Percentages show the distribution for each gender.

Table 8. Detailed age-related distribution of elastic ligature color preferences.*

\begin{tabular}{l|c|c|c}
\hline Color & $\leq 15$ & $16-20$ & $\geq 21$ \\
\hline Fire red & $17(8.6 \%)$ & $7(3.1 \%)$ & $0(0.0 \%)$ \\
\hline Lilac & $10(5.2 \%)$ & $16(7.0 \%)$ & $3(4.0 \%)$ \\
\hline Yellow & $5(2.5 \%)$ & $6(2.6 \%)$ & $0(0.0 \%)$ \\
\hline Blue & $16(8.1 \%)$ & $16(7.0 \%)$ & $1(1.3 \%)$ \\
\hline Pink & $36(18.3 \%)$ & $19(8.3 \%)$ & $4(5.3 \%)$ \\
\hline Lime & $10(5.2 \%)$ & $9(3.9 \%)$ & $1(1.3 \%)$ \\
\hline Purple & $5(2.5 \%)$ & $4(1.8 \%)$ & $2(2.7 \%)$ \\
\hline Black & $10(5.1 \%)$ & $10(4.4 \%)$ & $0(0.0 \%)$ \\
\hline Light Blue & $17(8.6 \%)$ & $13(5.8 \%)$ & $4(5.3 \%)$ \\
\hline Transparent & $43(21.8 \%)$ & $91(39.9 \%)$ & $50(66.8 \%)$ \\
\hline White & $4(2.0 \%)$ & $11(4.8 \%)$ & $4(5.3 \%)$ \\
\hline Pearl & $1(0.5 \%)$ & $3(1.3 \%)$ & $0(0.0 \%)$ \\
\hline Silver & $7(3.6 \%)$ & $7(3.1 \%)$ & $3(4.0 \%)$ \\
\hline Various & $16(8.0 \%)$ & $16(7.0 \%)$ & $3(4.0 \%)$ \\
\hline
\end{tabular}

* Percentages show the distribution for each age group. ful ligatures. The preference for less-noticeable elastic ligatures showed a gradual increase with increasing age: $27.9 \%$ in subjects aged less than 16 years, $49.1 \%$ in subjects aged $16-20$ years, and $76.0 \%$ in subjects aged more than 20 years. Another noteworthy finding was that transparent ligatures were mainly preferred by all age groups. The preference percentages for transparent ligatures were $21.8 \%$ for subjects aged less than 16 years, $39.9 \%$ for subjects aged between $16-20$ years, and $66.8 \%$ for subjects aged more than 20 years. This high preference may be explained by the desire to make the fixed orthodontic appliance less visible or to camouflage the appliance. This preference can be considered to be influenced by peer pressure and the esthetic concerns associated with the use of metal brackets.

\section{CONCLUSIONS}

Female patients preferred red-purple-colored tones, while male patients preferred blue-blackcolored tones. Adolescents preferred colorful elastic ligatures, while older patients preferred less-noticeable elastic ligatures. A stock of $10-$ 12 colorful and less-noticeable elastic ligatures seems adequate for patient satisfaction.

\section{REFERENCES}

1. Mizrahi E, ed. Orthodontic Pearls. A selection of practical tips and clinical expertise. London: Taylor \& Francis; 2004.

2. Hurlbert AC, Ling Y. Biological components of sex differences in color preference. Curr Biol 2007;17:R623-625.

3. Alexander GM. An evolutionary perspective of sex-typed toy preference: pink, blue, and the brain. Arch Sex Behav 2003;32:7-14.

4. Ruble DN, Lurye LE, Zosuls KM. Pink frilly dresses (PFD) and early gender identity. Princeton Report of Knowledge, Volume 2(2), Princeton University. Available from: http:// www.princeton.edu/prok/issues/2-2/pink_frilly.xml. 